\title{
Investigation of flame propagation in autoignitive blends of n-heptane and methane fuel
}

\author{
ARTICLE HISTORY \\ Compiled April 26, 2019
}

\begin{abstract}
The effects of pre-ignition chemistry on laminar flame speed in methane/n-heptane fuel blends are investigated numerically, leading to flame speed modelling accounting for these effects. The laminar flame speeds of fuel blends are important input parameters for turbulent combustion models needed to support design of dual-fuel engines. At the autoignitive conditions found in engines, pre-ignition reactions cause the speed of the reaction front to increase. Fuels that exhibit two-stage ignition behaviour, such as n-heptane, also exhibit a two-stage increase in the speed of the reaction front as the reactant residence time increases. There is a corresponding reduction in the flame thickness until the residence time approaches the ignition delay time, whereupon the deflagrative scaling of flame thickness breaks down. The analysis shows that the increase in flame speed is due to distinct contributions of heat release, reactant consumption, and enhanced reactivity ahead of the flame. Addition of methane to n-heptane-air mixtures retards and reduces the first-stage increase in flame speed, in part due to dilution of the more-reactive n-heptane fuel, and in part due to consumption of radical species by the methane chemistry. The effect of methane/n-heptane fuel blending on flame speed is described adequately by a linear mixing rule. The effect of pre-ignition chemistry can then be modelled as a linear function of the progress variable ahead of the flame - accounting for heat release, reactant consumption, and enhanced reactivity ahead of the flame. The flame speed model accurately describes the variation of flame speed across the full range of methane/n-heptane blends at engine-relevant conditions, up to the deflagration/ignition transition.
\end{abstract}

\section{KEYWORDS}

cool-flame; low temperature chemistry; two-stage ignition; residence time; flame speed; dual-fuel

\section{Introduction}

High efficiency combustion engines require conditions at which the reactants may become autoignitive. Even in engine systems nominally characterised by deflagrative combustion, flame propagation may still be affected by the pre-ignition chemical reactions taking place ahead of the flame. Such reactions can affect flame stability and prompt flashback in steady-flow combustors [1], and affect ignitability [2], heat release rates [3] and detonation phenomena [4] in piston engines. Ignition chemistry differs markedly among fuels, with some heavier hydrocarbon fuels exhibiting two-stage ignition under some conditions [5], leading to differences in the structure and speed of flame propagation through autoignitive mixtures [6]. These differences may be particularly relevant in dual fuel engines in which fuels with dissimilar ignition behaviours, such as natural gas and diesel, are introduced separately [7]. In pilot-ignited dual fuel 
engines, following the formation of ignition kernels, a majority of the pilot fuel may be consumed in polybrachial flame structures led through the dual-fuel mixture by deflagrative edge flames that propagate along stoichiometric isosurfaces [8]. However the effect that blending fuels with dissimilar ignition chemistry has on flame propagation through autoignitive mixtures is not well characterised.

Hydrogen and light hydrocarbon fuels such as methane exhibit single-stage hightemperature ignition behaviour. Pre-ignition reactions have limited effect on flame propagation through single-stage ignition mixtures until the mixture is close to the point of autoignition $[6,9,10]$. In contrast, some heavier hydrocarbons relevant to real transport fuels, such as n-heptane, exhibit low temperature chemistry (LTC) [11] and cool flames [12]. The chemical kinetics controlling cool flames arise at high pressure and moderate temperatures, and are closely related to those of two-stage ignition and Negative Temperature Coefficient (NTC) behaviour [5, 13]. Following much of the literature, the term 'cool flame' is used indiscriminately here to refer both to deflagrative and to ignitive reaction fronts involving low temperature chemistry. Low temperature chemistry can significantly modify the chemical and transport properties of a mixture [3], thereby affecting the laminar flame speed in mixtures well before the onset of high-temperature ignition $[6,14]$. The response of laminar flame properties to blending of fuels with dissimilar ignition behaviours, such as methane and n-heptane, has not been characterised fully at autoignitive conditions. Methane/n-heptane mixtures are of particular interest because these have been used in research studies as surrogate fuels for the natural gas/diesel mixtures arising in pilot-ignited dual fuel engines, (e.g., $[15-17])$.

The laminar flame speed and laminar flame thickness are centrally important in characterisation and modelling of turbulent combustion in engines because they directly affect the turbulent flame speed [18]. The dependence of the turbulent flame speed on laminar flame properties persists for flames affected by LTC $[1,3]$. Knowledge of the laminar flame speed and laminar flame thickness are therefore key to understanding and modelling turbulent combustion processes under the autoignitive conditions that predominate in practical combustion systems. However established empirical models for the variation of flame speeds with temperature, pressure, equivalence ratio and dilution, such as in Ref. [19], do not account for the effect of pre-ignition chemical processes on flame speed.

\subsection{Laminar flame speed}

Pre-ignition chemical reactions ahead of a flame affect the propagation speed. As such, there is not a unique freely-propagating laminar flame speed $\left(s_{l}\right)$ at autoignitive conditions. Rather, the flame speed depends on the extent of the pre-ignition reactions ahead of the flame $[6,9,14]$, which can be related to the residence time $\tau_{f}[20,21]$ between the reactants mixing and arriving at the flame front. The propagation speed of the flame front can be evaluated in a general way from the density-corrected displacement speed $s_{f}[22]$. Evaluating $s_{f}$ in any unstrained one-dimensional stationary flame configuration yields a well-defined residence time-dependent flame speed, provided that diffusive fluxes through the inlet of the flow domain are negligible [6].

A non-autoignitive freely-propagating laminar flame corresponds to the limit where the residence time is much less than the ignition delay time $\tau_{i g n}$, i.e. $\tau_{f} \ll \tau_{i g n}$. Conversely, the limit $\tau_{f} \rightarrow \tau_{i g n}$ corresponds to the transition from a deflagrative flame to a pure ignition front [23]. Hereafter we refer to the flame speed in the short residence 
time limit $\tau_{f} \ll \tau_{i g n}$ as reference flame speed $s_{r}[6]$.

\subsection{Laminar flame thickness}

In the case of diffusion-limited flame propagation with negligible chemical reaction upstream of and within the preheat layer, the thermal thickness of the flame front $\delta_{f}$ scales with the thermal diffusivity $\alpha$ and laminar flame speed as [24]

$$
\delta_{f} \sim \frac{\alpha}{s_{f}}
$$

The thermal thickness is $\delta_{f}=\Delta T /(d T / d x)_{\max }$, where $\Delta T$ is the temperature rise across the front and $(d T / d x)_{\max }$ is the maximum temperature gradient within the flame. Since the variation of thermal diffusivity and flame speed with reactant properties are typically well-modelled by established empirical relations, (e.g., [19]), this scaling relationship provides a simple means for estimating how the reaction front thickness varies across a range of combustion conditions for which the flame front behaviour is deflagrative. This scaling relationship breaks down as the flame transitions into an ignition front. The transport equation for progress variable $c$ within a stationary ignition front is

$$
u \frac{\partial c}{\partial x}=\frac{\omega_{c}}{\rho},
$$

where $x$ and $u$ are the displacement and velocity normal to the flame and $\omega_{c}$ is the reaction source term for progress variable. The thermal thickness of the reaction front can be approximated using the progress variable gradient: $\delta_{f} \sim(\partial c / \partial x)_{\max }^{-1}$, giving the relationship

$$
\delta_{f} \sim \frac{\rho_{u}}{\omega_{c, \max }} s_{f}
$$

in which the factor $\rho_{u} / \omega_{c, \max }$ is positive, with magnitude dependent on the thermochemical state of the reactants. The relationship between $\delta_{f}$ and $s_{f}$ therefore provides an indication of whether a flame front is deflagrative $(\sim$ Eq. 1$)$ or ignitive $(\sim$ Eq. 3).

The objectives of this study are to investigate and to model the effects of different methane/n-heptane fuel blends and their pre-ignition chemistry on the speed of reaction fronts under dual-fuel compression-ignition engine conditions. The following section sets out the physical modelling and numerical approach adopted. The results are then analysed in order (1) to assess the effects on flame speed and structure due to thermal and chemical contributions of the different fuels; and (2) to develop a new modelling approach for reaction front speeds under autoignitive conditions.

\section{Methodology}

Simulations of adiabatic un-stretched steady one-dimensional laminar flames are used to investigate the combined effects of methane/n-heptane ratios and pre-ignition chemistry at engine-relevant temperatures. The effect of the pre-ignition chemistry is assessed by varying the residence time upstream of the flame front. The residence time 


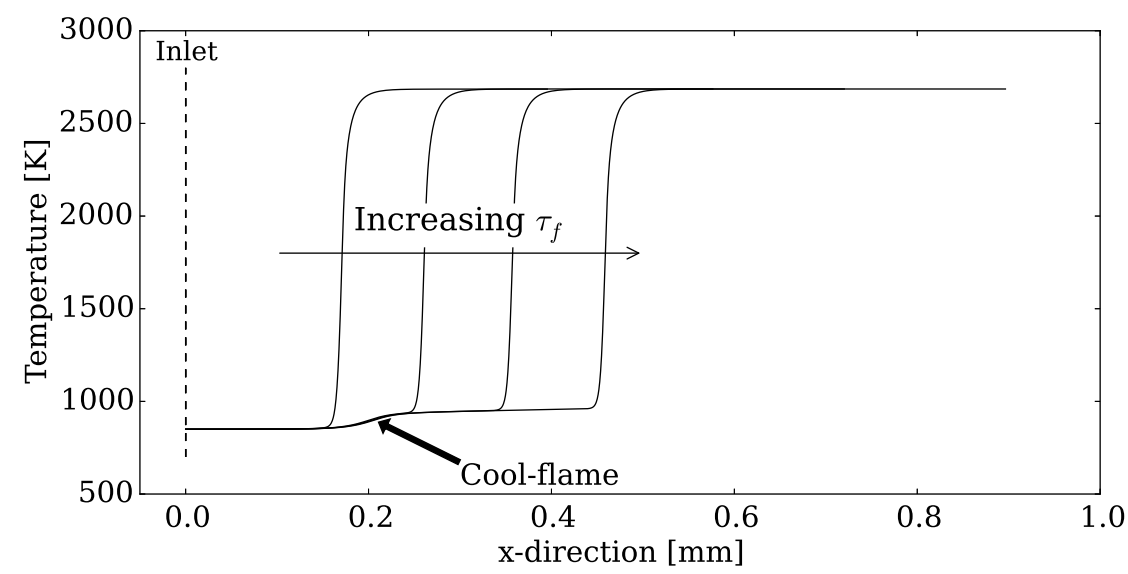

Figure 1. The temperature profile in several steady freely-propagating stoichiometric n-heptane-air flames with different residence times at $850 \mathrm{~K}$ and 40 bar. The location of the cool flame front is indicated.

is changed by varying the position specified for the flame relative to the inlet of the solution domain, as illustrated in Fig. 1. The residence time $\tau_{f}$ at the flame front is evaluated as

$$
\tau_{f}=\int_{x_{i}}^{x_{f}} \frac{1}{u(x)} d x
$$

where $x_{i}$ is the $x$-location of the inlet to the solution domain, $x_{f}$ is the location of the upstream edge of the flame, here defined as being half of one thermal thickness upstream of the maximum temperature gradient location.

Reactant mixtures are described in terms of their total-equivalence ratio, $\phi_{t o t}$, evaluated in the conventional manner by considering the stoichiometric oxygen-fuel ratio for the fuel mixture, and fuel-equivalence ratios $\phi_{C H_{4}}$ and $\phi_{C_{7} H_{16}}$ [25]. The fuel equivalence ratios are defined by $\phi_{C H_{4}}=\nu_{C_{4}} Y_{\left(C_{4}, u\right)} / Y_{\left(O_{2}, u\right)}$ and $\phi_{C_{7} H_{16}}=$ $\nu_{C_{7} H_{16}} Y_{\left(C_{7} H_{16}, u\right)} / Y_{\left(O_{2}, u\right)}$, where subscript $u$ denotes the unburnt composition and $\nu_{\text {fuel }}$ is the stoichiometric oxygen-to-fuel mass ratio for each fuel. These definitions of the fuel equivalence ratio can be added to obtain the total equivalence ratio, $\phi_{\text {tot }}=\phi_{\mathrm{CH}_{4}}+\phi_{\mathrm{C}_{7} \mathrm{H}_{16}}$.

The flames are simulated using the COSILAB one-dimensional flame solver [26] with multi-component molecular transport, ideal gas models with seven-coefficient polynomials for the temperature-dependence of thermodynamic properties. The methane/nheptane chemistry is modelled using a 106 species mechanism for n-heptane combustion [27], presenting a good compromise between accuracy and computational time. There are few experimental data available for autoignition and flame propagation in methane/n-heptane fuel blends at engine-relevant conditions. Therefore the chemical model has been selected on the basis of satisfactory autoignition and flame propagation predictions of experimental data for pure methane-air and n-heptane-air mixtures, and satisfactory agreement with more detailed models for autoignition and flame propagation in methane/n-heptane fuel blends. Validation data for the 106 species mechanism used are provided as supplementary material. Figure 2 presents the variation of ignition delay time with temperature for four stoichiometric methane/n-heptane fuel blends $\left(\phi_{C H 4} / \phi_{t o t}=0,0.5,0.8\right.$, and 1.0) in air at 40 bar, with results for the 106 species skeletal mechanism in reasonably close agreement with its 451 species parent 
detailed mechanism [28]. Fuel blends are mixed with air throughout this study, and air is modelled as $79 \% \mathrm{~N}_{2}$ and $21 \% \mathrm{O}_{2}$ by volume. The predictions show significant NTC behaviour at the conditions investigated in this study, with the NTC behaviour reducing as the proportion of methane in the fuel is increased.

The COSILAB software employs adaptive grid refinement and a stationary flame solution is obtained using a modified-Newton method [26]. The grid-independence of the solution data presented in this paper has been established by incrementally tightening the adaptive grid error tolerances until numerical convergence is achieved, requiring between one hundred and four hundred grid points, depending on the simulation conditions.

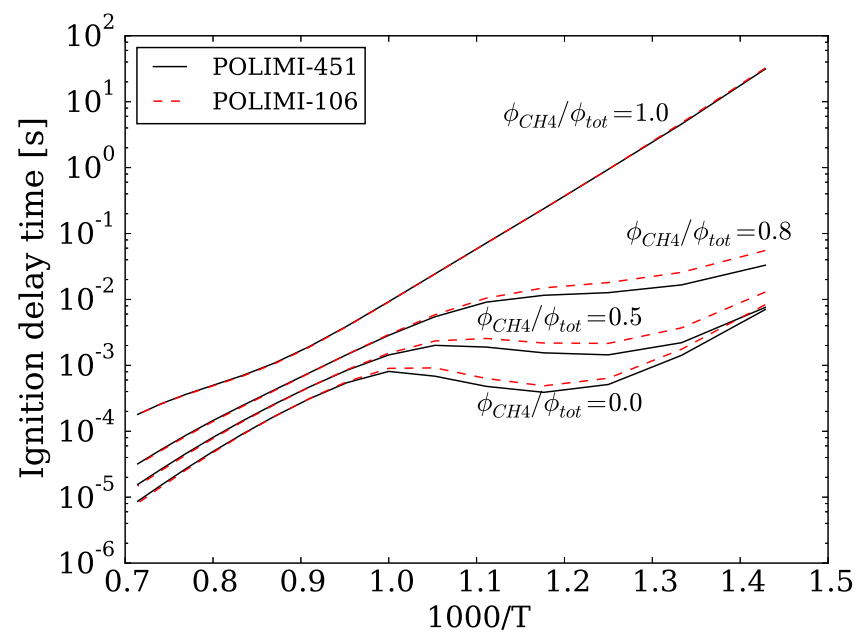

Figure 2. The temperature-dependence of ignition delay time for stoichiometric methane/n-heptane-air blends at 40 bar with $\phi_{C H_{4}} / \phi_{\text {tot }}=0.0,0.5,0.8,1.0$ computed with POLIMI-106 [27] and POLIMI-451 [28] mechanisms.

\section{Results and Discussion}

\subsection{Effects of pre-ignition chemistry on flame speed}

Figure 3 shows the variation of flame speed with residence time for pure methane, pure n-heptane and two methane/n-heptane fuel blends with global stoichiometric equivalence ratio $\left(\phi_{\text {tot }}=1\right)$ at 40 bar and 850 or $1000 \mathrm{~K}$. The results are presented in terms of residence time, rather than in terms of the flame positions reported in Refs. $[6,9]$, since, for autoignitive conditions, the chemical evolution upstream of the flame can be related to residence time, as shown below. The residence time may serve as a useful parameter for accounting for chemical evolution in a range of flows [21], whereas interpretation of the flame position is configuration specific. Nonetheless, the residence time and flame position are closely related in the present one-dimensional configuration using to Eq. 4. The flame speed of the methane-air mixture remains approximately constant until the residence time approaches the ignition delay time, when it increases indefinitely, as observed previously in Refs. [9, 10]. For an unburned temperature of $1000 \mathrm{~K}$ where n-heptane-air mixtures exhibit single-stage ignition, the evolution of the flame speed of the n-heptane-air mixture is qualitatively similar to the pure methane case. However, for an unburned temperature of $850 \mathrm{~K}$ where n-heptane-air mixtures 


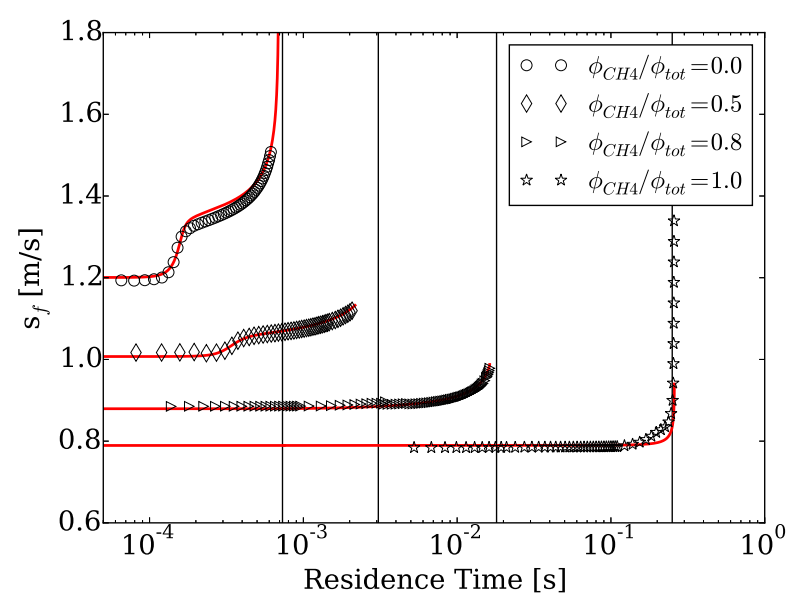

(a) $850 \mathrm{~K}$

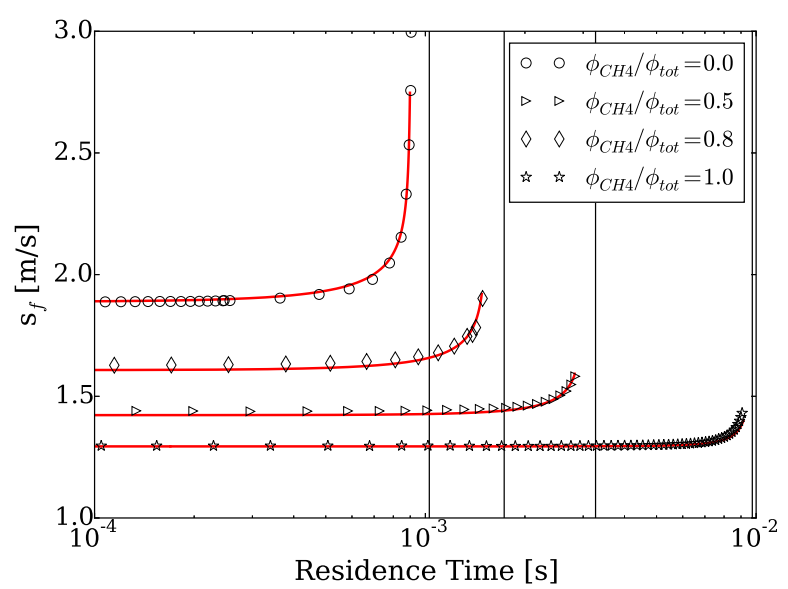

(b) $1000 \mathrm{~K}$

Figure 3. Laminar flame speeds of stoichiometric methane/n-heptane blends versus residence time at 40 bar: simulations (symbols); model Eq. 8 (solid).

exhibit two-stage ignition, the flame speed also increases in two distinct stages: the flame speed increases by $15 \%$ when the residence time reaches the first-stage ignition delay time, then increases gradually during the second-stage ignition-delay, before increasing indefinitely as the residence time approaches final ignition delay time. The same two-stage increase in flame speed is observed for methane/n-heptane blends that show two-stage ignition behaviour, and the two-stage effect is stronger as the proportion of n-heptane increases.

Figure 4 shows that the first-stage increase in flame speed coincides with the firststage increase in temperature, occurring between $0.13-0.18 \mathrm{~ms}$ residence time for 40 bar $850 \mathrm{~K}$ stoichiometric n-heptane-air flames. The flame speed is expected to be affected by the temperature increase, however consumption of major reactants and production of intermediate species by the pre-ignition chemical reactions ahead of the flame also affect flame speed. We conduct a numerical experiment in order to quantify the relative influence that the thermal and chemical changes have on the flame speed during first-stage ignition. The experiment isolates the effect of the intermediate species from the thermal effects by performing modified flame simulations in which we remove all intermediate species a short distance upstream of the flame while keeping 


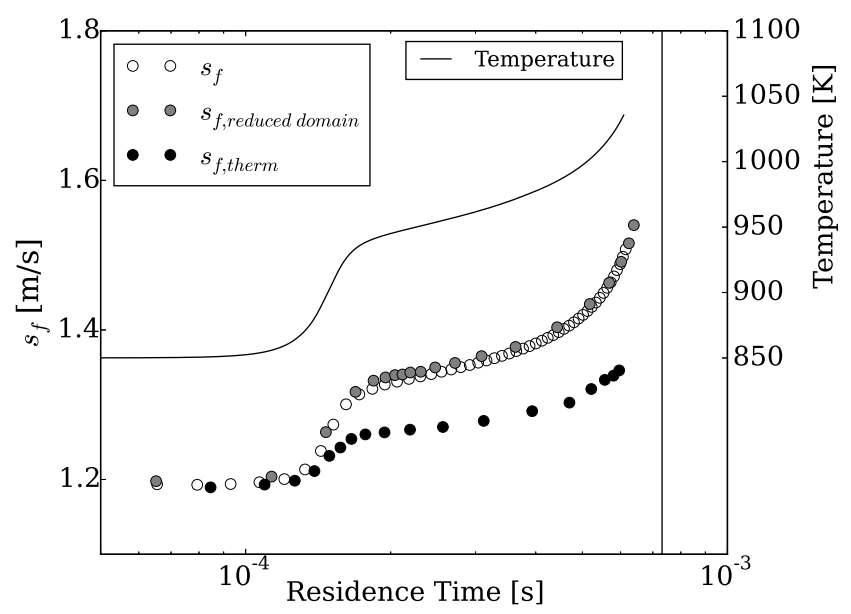

Figure 4. Laminar flame speed and temperature versus residence time for stoichiometric n-heptane-air at 40 bar and $850 \mathrm{~K}$ : Unmodified flame in the full domain (white circles); $0.2 \mathrm{~mm}$ domain with inlet composition from the unmodified flame (grey circles); $0.2 \mathrm{~mm}$ domain with intermediate species removed from the inlet composition (black circles).

the temperature and mixture enthalpy unchanged following the procedure set out in the Appendix, resulting in a modified flame speed $s_{f, t h e r m}$. Since the intermediate species have been removed from the modified flame, the variation of $s_{f, t h e r m}$ shown in Fig. 4 is entirely due to the temperature rise and the consumption of major reactants ahead of the flame front. The results of the modified flame experiment indicate that the first stage increase in flame speed is due to both thermal and chemical influences in approximately equal measure. Modelling for the flame speed should take each of these effects into account.

\subsection{Effects of pre-ignition chemistry on flame thickness}

Diffusion-limited (i.e. deflagrative) flame propagation is expected to exhibit a scaling relationship between flame thickness and flame speed given by Eq. 1. Figure 5 shows the variation of the high-temperature flame's speed with $\alpha / \delta_{f}$ for premixed combustion of stoichiometric methane-air and n-heptane-air over a range of reactant temperature $\left(T_{u}=700\right.$ to $\left.1000 \mathrm{~K}\right)$. Data are normalised by the reference laminar flame speeds of methane $\left(s_{r, 0 C H_{4}}=0.79 \mathrm{~ms}^{-1}\right)$ and n-heptane $\left(s_{r, 0 C_{7} H_{1} 6}=1.20 \mathrm{~ms}^{-1}\right)$ at $T_{0}=850 \mathrm{~K}$ and $p_{0}=40$ bar, where subscript ' 0 ' refers to datum conditions. Reference properties for flames with short residence times (shown as symbols) follow the deflagrative scaling relationship as $T_{u}$ is increased from 700 to $1000 \mathrm{~K}$. The gradient of $s_{r}$ with respect to $\alpha / \delta_{r}$ is greater for n-heptane-air flames due to the greater reactivity of n-heptane compared to methane. As residence time increases (dashed lines), the flame speed of the n-heptane-air mixture remains largely unchanged until the mixture undergoes first-stage ignition. Subsequently the reaction front speed increases, and the reaction front thickness initially reduces in accordance with the deflagrative scaling given in Eq. 1. Finally, as the temperature-based progress variable $\left(c \equiv\left(T-T_{u}\right) /\left(T_{b}-T_{u}\right)\right)$ ahead of the flame reaches around 0.15 , at the locations indicated by square symbols in Fig. 5, the deflagrative scaling breaks down and the front thickness starts to increase $\left(\alpha / \delta_{f}\right.$ reduces because the residence time ahead of the flame approaches the ignition delay time - marking the transition from deflagrative to ignitive scaling of the front 


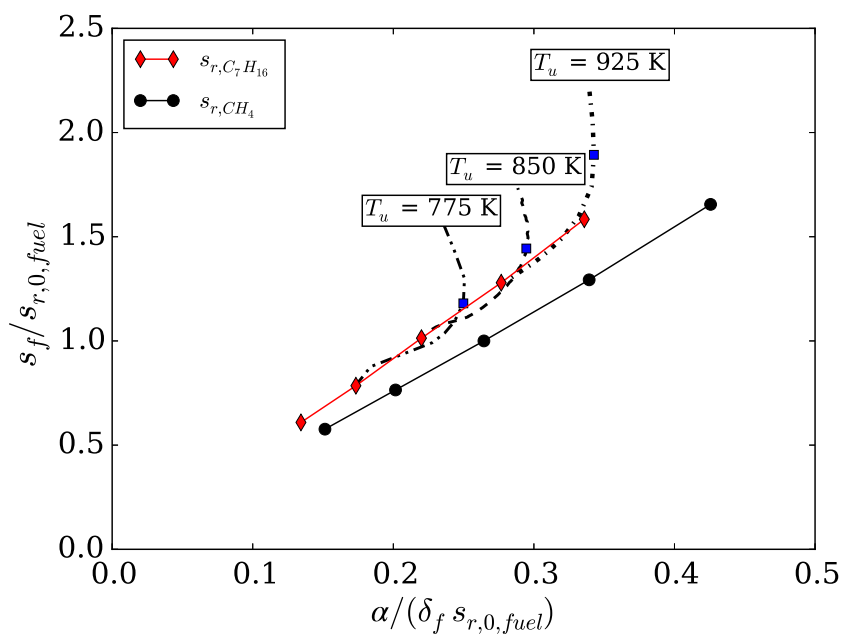

Figure 5. Normalised high-temperature reaction front speed $s_{f} / s_{r, 0, \text { fuel }}$ versus $\alpha /\left(\delta_{f} s_{r, 0, f u e l}\right)$ for short residence time $\left(\tau_{f} \longrightarrow 0\right)$ stoichiometric methane-air and n-heptane-air flames with unburnt temperatures $700,775,850,925$ and $1000 \mathrm{~K}$ (symbols), and data for n-heptane-air combustion with residence times up to $98 \%$ of the ignition delay time at 775,850 , and $925 \mathrm{~K}$ (various dashed lines as labelled). Square symbols indicate the conditions where the progress variable ahead of the flame equals 0.15 .

\section{thickness.}

Figure 6 presents the variation of the cool-flame's speed and thickness for the $850 \mathrm{~K}$ 40 bar stoichiometric n-heptane-air flame as the residence time increases. The thickness of the cool-flame follows the linear scaling behaviour given for ignition fronts in Eq. 3. Despite its name, the cool-flame exhibits ignitive rather than deflagrative behaviour across all of the conditions in this study: the data flow the a linear relationship between cool-flame speed and thickness as indicated by the dashed lines in Fig. 6. The absence of deflagrative cool-flames is consistent with Ref. [29], where steady deflagrative cool-flames could only be stabilised when aerodynamic straining was used to prevent the development of a high-temperature flame. Ju et al. [30], in contrast, showed that steady deflagrative double-flame structures, involving a cool-flame and a hot-flame, can arise under very lean low-temperature combustion conditions (e.g. $\phi=0.1, T_{u}=530 \mathrm{~K}$ ). The combustion conditions investigated by Ju et al. are unlike the conditions in most engines since the magnitude of heat release in the cool-flame is similar to the magnitude of heat release in the hot flame. At richer, higher-temperature conditions typical of current engines, it may be that the magnitude of heat release in the hot-flame is greater, such that its flame speed would cause it to over-take any cool-flame, should a cool-flame arise, preventing observation of steady deflagrative cool-flames.

The transition between deflagrative and ignitive scaling of the flame thickness is accompanied by diminishing importance of diffusive transport within the flame fronts. Figure 7 shows mass fraction transport budgets for stoichiometric n-heptane flames at $850 \mathrm{~K}$ and 40 bar for two residence times $\tau_{f} / \tau_{i g n}=0.85$ and 0.95 . The reaction $(\mathrm{R})$, convection (C) and diffusion (D) terms [6] are presented for the alkyl hydroperoxy radical $\left(\mathrm{C}_{7} \mathrm{H}_{1} 5 \mathrm{OOH}\right.$, abbreviated to $\left.\mathrm{QOOH}\right)$ within the cool-flame and for $\mathrm{OH}$ within the high-temperature flame front. The convection term arises because the simulated flames are stationary. The budgets indicate that diffusive transport in the high-temperature reaction front becomes gradually less important as the residence time increases, and is generally less important in the cool-flame front. The magnitude of the diffusion term 


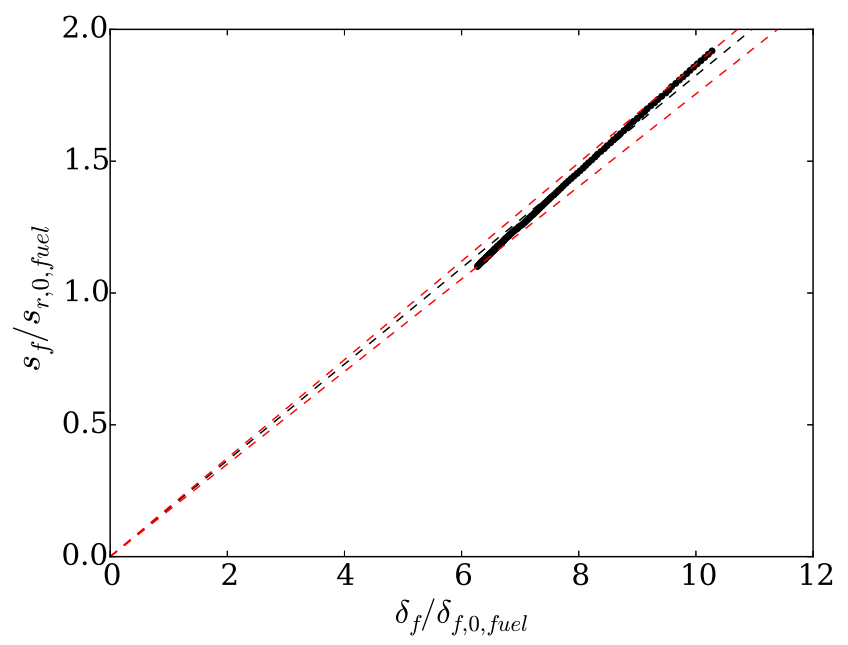

Figure 6. Cool-flame reaction front speed $s_{f}$ versus thermal thickness $\delta_{f}$ for stoichiometric n-heptane-air flames normalised by reference flame properties at $850 \mathrm{~K}$ and 40 bar for a range of residence times (symbols). Data range from $\tau_{f}=1.6 \cdot 10^{-04} \mathrm{~s}$ to $\tau_{f}=7.1 \cdot 10^{-04} \mathrm{~s}$. Dashed lines correspond to Eq. 3.

in the high-temperature flame is lower at $\tau_{f} / \tau_{i g n}=0.95$, at which point the flame thickness is increasing with flame speed, than at $\tau_{f} / \tau_{i g n}=0.85$, at which point the flame thickness is reducing in accordance with the deflagrative scaling (Eq. 1). However the gradual reduction of the diffusive transport contribution does not provide as clear a delineation of the transition between deflagrative and ignitive behaviours as the changes in the $s_{f}-\delta_{f}$ dependence shown in Figs. 5 and 6 .

Analysis of the flame thickness indicates that the deflagrative scaling in Eq. 1 applies to the high-temperature flame front across a wide range of autoignitive conditions and, given models for $s_{f}$ and $\alpha$, provides a useful means of modelling the variation of $\delta_{f}$ in a flow up to the transition to ignitive behaviour.

\subsection{Flame speeds at autoignitive conditions}

The laminar flame speed is a key input for a number of turbulent combustion models [18]. For a given set of conditions the reference laminar flame speed may be evaluated from experimental measurements or, if suitable chemical and thermodynamic models are available, from a laminar flame simulation. The laminar flame speed may be provided to a turbulent combustion model by means of a data table or some form of algebraic fit to the data. Several empirically-derived algebraic models for laminar flame speed have been developed and used widely for combustion of individual fuels at non-autoignitive conditions [19]. Furthermore, due to the effort required to obtain flame speed data across a wide range of operating conditions as well as for different blends of fuels, mixing rules have been proposed in order to estimate the reference laminar flame speed of blends of individual fuels for which reference flame speeds are available [31-33]. For combustion under autoignitive conditions, however, the preceding analysis indicates that it is necessary also to account for the effect of pre-ignition chemistry on the flame speed, including the distinct contributions of heat release, reactant consumption and enhanced reactivity ahead of the flame. Due to the large domain size needed for the simulations required to generate the data presented in Fig. 3 it is not practical to compute the flame speed directly for the full range of residence 

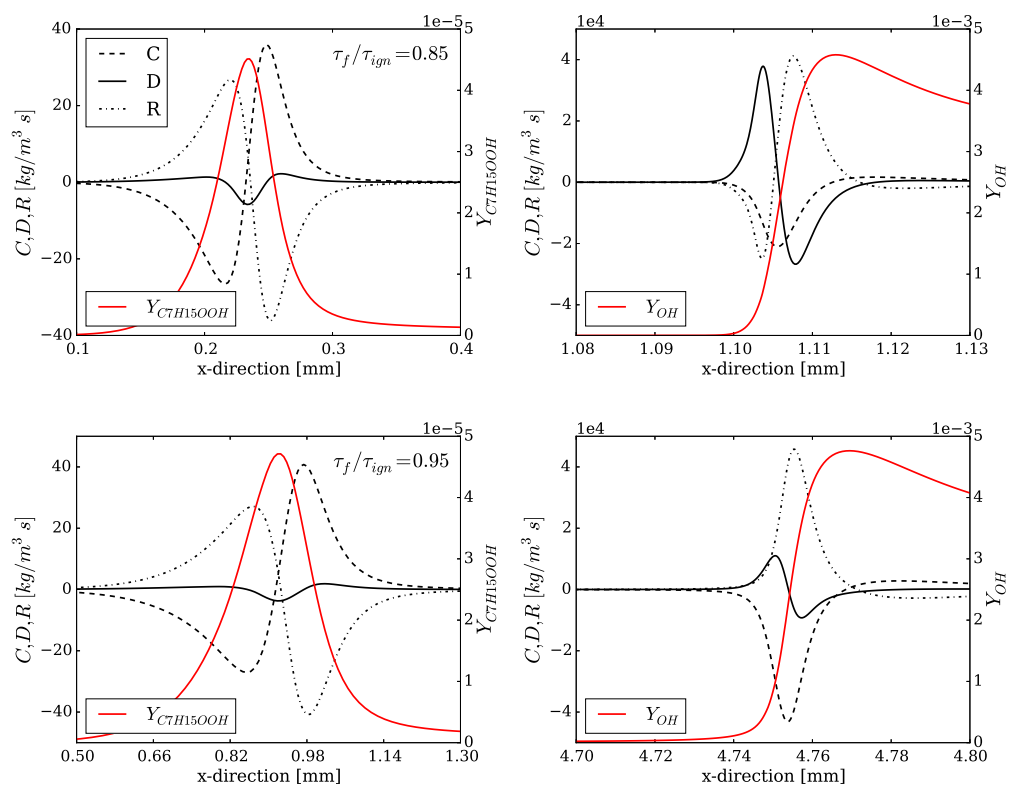

Figure 7. Transport budgets and mass fraction profiles for stoichiometric n-heptane-air at $850 \mathrm{~K}$ and 40 bar for $Y_{Q O O H}$ (left column) and $Y_{O H}$ (right column). The residence times at the flame front are $85 \%$ (top row), and $95 \%$ (bottom row) of the overall ignition delay time. The data are plotted versus the distance from the inlet.

times, fuel blends and mixture conditions that arise during operation of a dual-fuel engine. The objective of this section therefore is to develop a method for estimating the effects of pre-ignition chemistry on flame speed taking the reference flame speed as an input. This novel method can be applied to reference flame speeds obtained by any means.

In order to evaluate reference flame speeds efficiently and accurately in this study, well-established algebraic models are used to account for the dependence on equivalence ratio and the blending between fuels [31].

Yang and Reitz [31] modelled the dependence of reference flame speed $s_{r}$ on equivalence ratio using a four-parameter Gaussian function and accounted for fuel blending using a linear mixing rule which, for blends of methane and n-heptane, takes the form,

$$
s_{r, m i x}=Z_{C H_{4}} s_{r, C H_{4}}+\left(1-Z_{C H_{4}}\right) s_{r, C_{7} H_{16}}
$$

where $Z_{C_{H_{4}}}=Y_{C_{H_{4}, u}} /\left(Y_{C_{H_{4}, u}}+Y_{C_{7} H_{16}, u}\right)$ is the local mass fraction of methane in the fuel blend. The Gaussian fit for methane and n-heptane flame speeds, and the flame speeds of methane/n-heptane blends given by Eq. 5 are validated for a range of equivalence ratio in Fig. 8, showing a close fit to the numerically-obtained flame speeds. Alternative mixing rules by Hirasawa et al. [32] and Di Sarli et al. [33] are also tested in Fig. 8. The Di Sarli et al. model tends to over-emphasise the contribution of methane. The linear model is adopted here as it is simpler and marginally more accurate than the Hirasawa et al. model for methane/n-heptane combustion cases considered. 


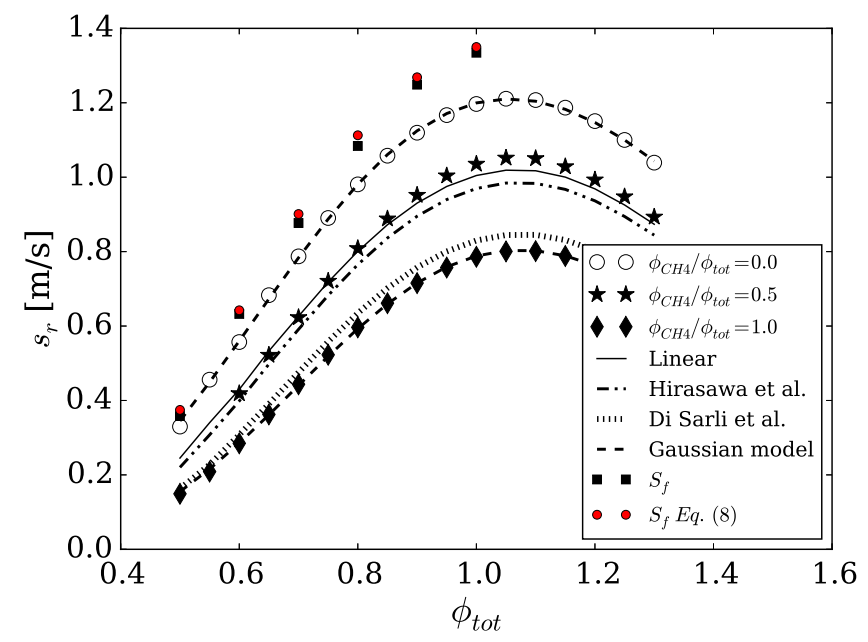

Figure 8. Variation of flame speed with equivalence ratio for methane/n-heptane fuel blends at $850 \mathrm{~K}$ and 40 bar for $\phi_{C H 4} / \phi_{\text {tot }}=0,0.5$ and 1.0; and linear [31], Hirasawa et al. [32], and Di Sarli et al. [33] mixing-rules. Closed square and circular symbols correspond to flame speeds from simulatins and from Eq. 8 respectively for residence times between 40 and $60 \%$ of the ignition delay time

\subsection{Effects of pre-ignition chemistry}

The form of the Metghalchi and Keck model [19] suggests how one can account for the effects of the temperature rise and reactant consumption arising ahead of the flame at autoignitive conditions, providing the first two factors on the right hand side of the following equation:

$$
s_{f, f u e l}=s_{r, f u e l} \cdot\left(\frac{T}{T_{u}}\right)^{\alpha_{f u e l}} \cdot\left(1-\beta_{f u e l} \xi_{b}\right) \cdot \mathcal{F}(c) \cdot\left(\frac{\rho}{\rho_{u}}\right) .
$$

Eq. 6 accounts for the flame speed increase due to the temperature increasing by the factor $T / T_{u}$ ahead of the flame. The flame speed decreases by the factor $\left(1-\beta_{\text {fuel }} \xi_{b}\right)$ because some of the fuel and oxidiser are consumed by the pre-ignition reactions, diluting the mixture with mass fraction $\xi_{b}$ of burned products. $\alpha_{\text {fuel }}$ and $\beta_{\text {fuel }}$ are fuel-dependent model coefficients. Accounting for the thermal expansion upstream of a one-dimensional planar flame, the flame speed is multiplied by the density ratio $\rho / \rho_{u}$, where $\rho$ is the density just ahead of the flame. A further factor $\mathcal{F}(c)$ is introduced to account for the increase in flame speed due to the chemical influence of intermediate species produced upstream of the flame; this influence is shown by the difference between $s_{f}$ and $s_{f, \text { therm }}$ in Fig. 4 . Fig. 9 shows that $s_{f} / s_{f, \text { therm }}$ has an approximately linear dependence on progress variable. This suggests a simple model:

$$
\mathcal{F}(c)=1+\gamma_{\text {fuel }} . c,
$$

with $\gamma_{C_{7} H_{16}} \approx 1.18$ for n-heptane. Equation 6 thus provides a breakdown of the contributions to the increase of the flame speed under autoignitive conditions.

Figure 9 shows that, for small values of progress variable, the laminar flame speed at autoignitive conditions increases approximately linearly with the value of progress variable $c$ just ahead of the flame for the full range of methane/n-heptane fuel blends 


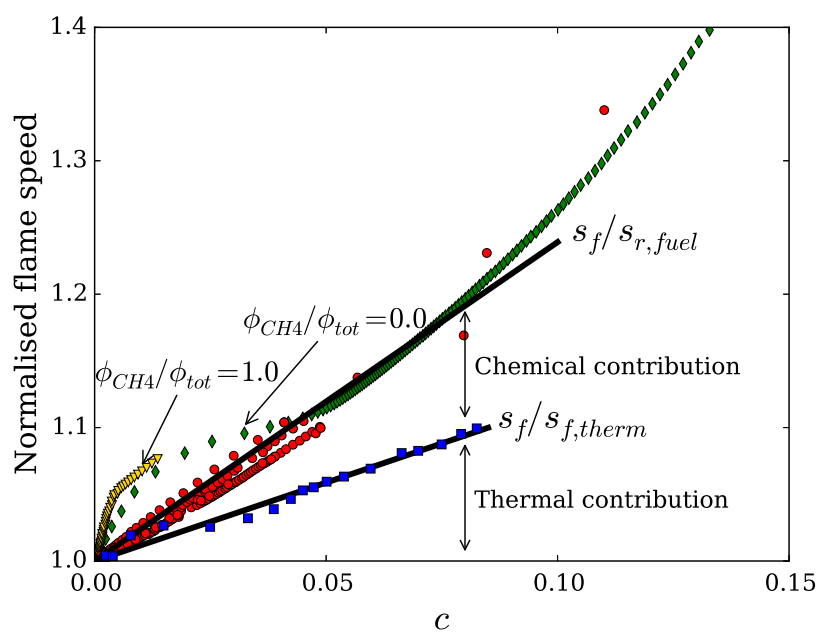

Figure 9. Normalised flame speed versus the progress variable upstream of the flame front: Round symbols show $s_{f} / s_{r, f u e l}$ at $\phi_{C H_{4}} / \phi_{\text {tot }}=0,0.5,0.8$ and 1.0 for $T_{u}=850 \mathrm{~K}$ and $1000 \mathrm{~K}$; Square symbols show $s_{f} / s_{f, \text { therm }}$ based on data from Fig. 4. Solid lines show $s_{f} / s_{r}=2.4 c$ and $s_{f} / s_{f, \text { therm }}=1.18 c$.

investigated, suggesting that Eq. 6 may be linearised into the form

$$
s_{f, f u e l}=s_{r, f u e l}\left(1+\epsilon_{f u e l} . c\right) .
$$

where $\epsilon_{f u e l}$ is a model coefficient. The data presented in Fig. 9 suggests that $\epsilon_{\text {fuel }}$ is in the range $2.4 \pm 0.5$ for the blends considered $\left(\phi_{C H_{4}} / \phi_{t o t}=0,0.5,0.8,1.0\right)$ for progress variable less than 0.1 . The linear model applies well to mixtures of methane and n-heptane, with pure n-heptane-air and methane-air blends some deviation from the linear model at $850 \mathrm{~K}$ for small values of progress variable, for which the increase in flame speed is anyway relatively small (shown by diamond and triangular symbols in Fig. 9). The value is potentially fuel specific and significantly higher values are obtained for example with dimethyl ether blends (not shown). From Eq. 6, the temperature-dependence leads to super-linear behaviour as the residence time approaches the ignition delay and the flame transitions into an ignition front, however this is of limited concern in the modelling of flame speed as flamelet-based turbulent combustion models that require flame speed as an input would not be applicable to flames with ignitive, rather than deflagrative behaviour. Therefore the linear modelling approach remains valid for the deflagrative combustion conditions at which the flamelet approach is applicable.

The model has been developed with respect to numerically-simulated flame speed data, therefore the model coefficient $\epsilon_{\text {fuel }}$ is subject to uncertainty associated with the accuracy of the chemical mechanism employed. However the broad validity of the relatively detailed 106 species mechanism used in this study suggests that the approximately-linear response of flame speed indicated in Fig. 9 is at least qualitatively correct. In principle, $\epsilon_{f u e l}$ could be evaluated directly from experimental measurement rather than by computation, however experimental measurements of flame speeds in partially-reacted mixtures are likely to be very challenging and will also involve significant, possibly greater, uncertainty.

The specification of progress variable may also affect the value of the model coefficient $\epsilon_{f u e l}$. Linear combinations of species mass fractions may be used as a basis for progress variable as an alternative to temperature, however it is important to employ 
a progress variable specification that resolves the evolution of the low-temperature combustion processes. For example, it is found that a progress variable specification based on the sum of carbon dioxide and carbon monoxide mass fractions also yields a linear flame-speed relationship, whereas use of carbon dioxide alone would not be appropriate since relatively little carbon dioxide is produced by the low-temperature chemistry. Due to the weak non-linearity between alternative definitions of progress variable, small differences in the value of $\epsilon_{f u e l}$ can arise depending on the progress variable specification.

In order to linearise Eq. 6, each of the factors in Eq. 6 can be expressed as a function of progress variable: rearranging the definition of progress variable gives $T / T_{u}=1+$ $c\left(T_{b} / T_{u}-1\right)$; approximating the fraction of reactants that have been consumed by the progress variable gives $\xi_{p r} \approx c$; and, neglecting changes in pressure and molar mass, the ideal gas equation gives $\rho / \rho_{u} \approx\left[1+c\left(T_{b} / T_{u}-1\right)\right]^{-1}$. Since the model is only required to be valid for small values of progress variable, a first-order Taylor expansion of Eq. 6 yields the linear relationship

$$
s_{f, \text { fuel }}=s_{r, f u e l}\left(1+\left[\left(\alpha_{\text {fuel }}-1\right) \times\left(\frac{T_{u}}{T_{b}}-1\right)-\beta_{\text {fuel }}+\gamma_{f u e l}\right] . c\right) .
$$

The coefficients $\alpha_{C_{7} H_{16}}=2.79, T_{u} / T_{b}=3.18, \beta_{C_{7} H_{16}}=3.02$ are obtained by leastsquares fitting to a data set containing reference flame speeds for $n$-heptane flames with $700<T_{u}<1000 \mathrm{~K}, 20<p<60$ bar, $0.8<\phi<1.2$ and $0<\xi<0.15$. These coefficients and $\gamma_{C_{7} H_{16}}=1.18$ lead to a prediction that $\epsilon_{C_{7} H_{16}}=2.1$, which is reasonably close to the value of $\epsilon \approx 2.4$ obtained from Fig. 9. Since the difference in $\epsilon_{f u e l}$ between the methane/n-heptane fuel blends considered is relatively small it is convenient to adopt a single value $\epsilon=2.4$ for the full range of fuel blends.

The flame speed model given by Eq. 8 is tested in Fig. 3, providing a good prediction of the variation of reference flame speed with residence time for all of the fuel blends and temperatures investigated. The model is tested across a range of lean equivalence ratios in Fig. 8, showing that the simple linear model correctly describes the reducing magnitude of the pre-ignition chemistry effect reduces under lean conditions. In order to apply the flame speed model in engine simulations, the progress variable in the mixture ahead of the flame then needs to be modelled, either by simulating the evolution of the chemical composition during ignition as in Ref. [34], or potentially by modelling the progress variable as a function of the Livengood-Wu integral [35].

\section{Conclusions}

The effects of pre-ignition chemistry on laminar flame speed in autoignitive methane/nheptane fuel blends are investigated using premixed laminar flame simulations. Preignition reactions cause the speed of the flame to increase. Fuels that exhibit two-stage ignition behaviour, such as n-heptane, also exhibit a two-stage increase in the speed of the reaction front as the reactant residence time increases. The increase in flame speed is due to distinct contributions of heat release, reactant consumption, and enhanced reactivity ahead of the flame. Addition of methane to n-heptane-air mixtures retards and reduces the first-stage increase in flame speed, in part due to dilution of the more-reactive n-heptane fuel, and in part due to consumption of radical species by the methane chemistry. As the residence time of the reactants approaches the ignition delay time, the reaction front transitions into a pure ignition front, in which diffusive 
transport is negligible.

Prior to transitioning into a pure ignition front, the behaviour of the flame can be classified as deflagrative or ignitive depending whether the flame thickness and flame speed obey the deflagrative scaling $\delta_{f} \sim \alpha / s_{f}$. The thickness of cool-flames exhibits ignitive scaling with flame speed, $\delta_{f} \sim s_{f}$, for all conditions simulated. The transition between deflagrative and ignitive scaling is also associated with a reduction in the relative magnitude of diffusive transport within the flame front, however examination of the transport budget alone does not provide a sharp delineation between deflagrative and ignitive behaviours.

Modelling for flame speed in dual-fuel blends at autoignitive conditions should account for the local composition of the fuel blend, and for the effects of pre-ignition chemistry. Modelling is introduced to account for the effects of pre-ignition chemistry on the flame speed. The model is a simple linear function of the progress variable ahead of the flame - accounting for the distinct contributions of heat release, reactant consumption, and enhanced reactivity ahead of the flame. The flame speed model accurately describes the variation of flame speed and hence flame thickness for the full range of methane/n-heptane blends at engine-relevant conditions, up to the deflagration/ignition transition.

\section{References}

[1] B. Windom, S.H. Won, C.B. Reuter, B. Jiang, Y. Ju, S. Hammack, T. Ombrello, and C. Carter, Study of ignition chemistry on turbulent premixed flames of n-heptane/air by using a reactor assisted turbulent slot burner, Combustion and Flame 169 (2016), pp. 19 -29 .

[2] G.A. Lavoie, J. Martz, M. Wooldridge, and D. Assanis, A multi-mode combustion diagram for spark assisted compression ignition, Combustion and Flame 157 (2010), pp. $1106-$ 1110.

[3] S.H. Won, B. Windom, B. Jiang, and Y. Ju, The role of low temperature fuel chemistry on turbulent flame propagation, Combustion and Flame 161 (2014), pp. $475-483$.

[4] Z. Wang, Y. Qi, X. He, J. Wang, S. Shuai, and C.K. Law, Analysis of pre-ignition to super-knock: Hotspot-induced deflagration to detonation, Fuel 144 (2015), pp. 222 - 227.

[5] F.L. Dryer, Chemical kinetic and combustion characteristics of transportation fuels, Proceedings of the Combustion Institute 35 (2015), pp. 117 - 144.

[6] A. Krisman, E.R. Hawkes, and J.H. Chen, The structure and propagation of laminar flames under autoignitive conditions, Combustion and Flame 188 (2018), pp. 399 - 411.

[7] T. Korakianitis, A.M. Namasivayam, and R.J. Crookes, Natural-gas fueled spark-ignition (SI) and compression-ignition (CI) engine performance and emissions, Progress in Energy and Combustion Science 37 (2011), pp. 89-112.

[8] A. Srna, M. Bolla, Y.M. Wright, K. Herrmann, R. Bombach, S.S. Pandurangi, K. Boulouchos, and G. Bruneaux, Effect of methane on pilot-fuel auto-ignition in dual-fuel engines, Proceedings of the Combustion Institute (2018).

[9] P. Habisreuther, F.C.C. Galeazzo, C. Prathap, and N. Zarzalis, Structure of laminar premixed flames of methane near the auto-ignition limit, Combustion and Flame 160 (2013), pp. $2770-2782$.

[10] J.B. Martz, H. Kwak, H.G. Im, G.A. Lavoie, and D.N. Assanis, Combustion regime of a reacting front propagating into an auto-igniting mixture, Proceedings of the Combustion Institute 33 (2011), pp. 3001-3006.

[11] F. Battin-Leclerc, Detailed chemical kinetic models for the low-temperature combustion of hydrocarbons with application to gasoline and diesel fuel surrogates, Progress in Energy and Combustion Science 34 (2008), pp. 440 - 498. 
[12] J.F. Griffiths and S.K. Scott, Thermokinetic interactions: Fundamentals of spontaneous ignition and cool flames, Progress in Energy and Combustion Science 13 (1987), pp. 161 $-197$.

[13] H.K. Ciezki and G. Adomeit, Shock-tube investigation of self-ignition of n-heptane-air mixtures under engine relevant conditions, Combustion and Flame 93 (1993), pp. 421 433.

[14] J. Pan, H. Wei, G. Shu, Z. Chen, and P. Zhao, The role of low temperature chemistry in combustion mode development under elevated pressures, Combustion and Flame 174 (2016), pp. $179-193$.

[15] S.K. Aggarwal, O. Awomolo, and K. Akber, Ignition characteristics of heptane-hydrogen and heptane-methane fuel blends at elevated pressures, international journal of hydrogen energy 36 (2011), pp. 15392-15402.

[16] S. Schlatter, B. Schneider, Y. Wright, and K. Boulouchos, Experimental study of ignition and combustion characteristics of a diesel pilot spray in a lean premixed methane/air charge using a rapid compression expansion machine, Tech. Rep., SAE Technical Paper, 2012.

[17] E. Demosthenous, G. Borghesi, E. Mastorakos, and R.S. Cant, Direct numerical simulations of premixed methane flame initiation by pilot n-heptane spray autoignition, Combustion and Flame 163 (2016), pp. 122-137.

[18] N. Peters, Turbulent Combustion, Cambridge Monographs on Mechanics, Cambridge University Press, 2000.

[19] M. Metghalchi and J.C. Keck, Burning velocities of mixtures of air with methanol, isooctane, and indolene at high pressure and temperature, Combustion and flame 48 (1982), pp. 191-210.

[20] D. Shin, R.D. Sandberg, and E.S. Richardson, Self-similarity of fluid residence time statistics in a turbulent round jet, Journal of Fluid Mechanics 823 (2017), pp. 1-25.

[21] D.H. Shin, E.S. Richardson, V. Aparece-Scutariu, Y. Minamoto, and J.H. Chen, Fluid age-based analysis of a lifted turbulent dme jet flame dns, Proceedings of the Combustion Institute (2018).

[22] F.A. Williams, The mathematics of combustion, SIAM, Philadelphia 97 (1985).

[23] Y.B. Zeldovich, Regime classification of an exothermic reaction with nonuniform initial conditions, Combustion and Flame 39 (1980), pp. 211 - 214.

[24] S.R. Turns, An introduction to combustion, 2000, MacGraw Hill, Boston, Massachusetts, US (2000).

[25] G.A. Karim, Dual-fuel diesel engines, CRC Press, 2015.

[26] Cosilab software, Tech. Rep., Cosilab, Rotexo Software, Bochum, 2011.

[27] E. Ranzi, A. Frassoldati, A. Stagni, M. Pelucchi, A. Cuoci, and T. Faravelli, Reduced kinetic schemes of complex reaction systems: fossil and biomass-derived transportation fuels, International Journal of Chemical Kinetics 46 (2014), pp. 512-542.

[28] E. Ranzi, A. Frassoldati, R. Grana, A. Cuoci, T. Faravelli, A.P. Kelley, and C.K. Law, Hierarchical and comparative kinetic modeling of laminar flame speeds of hydrocarbon and oxygenated fuels, Progress in Energy and Combustion Science 38 (2012), pp. 468-501.

[29] P. Zhao, W. Liang, S. Deng, and C.K. Law, Initiation and propagation of laminar premixed cool flames, Fuel 166 (2016), pp. $477-487$.

[30] Y. Ju, C.B. Reuter, and S.H. Won, Numerical simulations of premixed cool flames of dimethyl ether/oxygen mixtures, Combustion and Flame 162 (2015), pp. 3580-3588.

[31] S. Yang and R.D. Reitz, Integration of a continuous multi-component fuel evaporation model with an improved g-equation combustion and detailed chemical kinetics model with application to gdi engines, Tech. Rep., SAE Technical Paper, 2009.

[32] T. Hirasawa, C.J. Sung, A. Joshi, Z. Yang, H. Wang, and C.K. Law, Determination of laminar flame speeds using digital particle image velocimetry: binary fuel blends of ethylene, n-butane, and toluene, Proceedings of the Combustion Institute 29 (2002), pp. 1427-1434.

[33] V. Di Sarli and A. Di Benedetto, Laminar burning velocity of hydrogen-methane/air 
premixed flames, International Journal of Hydrogen Energy 32 (2007), pp. 637-646.

[34] B.S. Soriano, E.S. Richardson, S. Schlatter, and Y.M. Wright, Conditional moment closure modelling for dual-fuel combustion engines with pilot-assisted compression ignition, Tech. Rep., SAE Technical Paper, 2017.

[35] J.C. Livengood and P.C. Wu, Correlation of autoignition phenomena in internal combustion engines and rapid compression machines, in Symposium (International) on Combustion, Vol. 5. 1955, pp. 347-356.

\section{Appendix A. Procedure for removing intermediate species ahead of the flame}

The modified flame simulation involves two steps illustrated in Fig. A1.

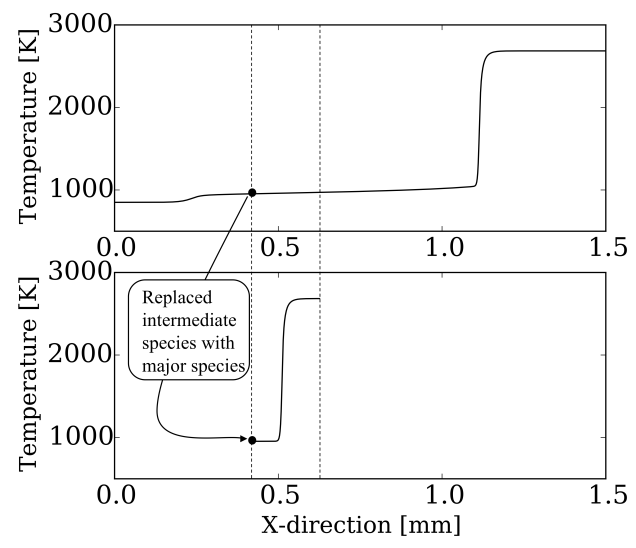

Figure A1. Illustration of the reduced-domain simulation procedure: temperature field from a full-domain simulation of a stoichiometric n-heptane-air flame with $\tau_{f}=0.95 \tau_{i g n}$ (top); temperature field from the reduceddomain simulation.

\section{A.1. Step 1}

The first step determines the modified composition ahead of the flame. A single precursor flame simulation (Fig. A1 top) is used to determine the variation of the reactant composition with residence time upstream of the flame front. The composition recorded for each residence time is then modified by replacing the intermediate species with a mixture of reactants and major products of stoichiometric combustion, while keeping the temperature and total enthalpy unchanged. The replacement mixture consists of $\mathrm{CH}_{4}, \mathrm{C}_{7} \mathrm{H}_{16}, \mathrm{O}_{2}, \mathrm{~N}_{2}, \mathrm{CO}_{2}$, and $\mathrm{H}_{2} \mathrm{O}$. The modified mass fraction vector $\mathbf{Y}^{\prime}$ is calculated by weighting the unburnt and burnt composition of complete combustion, $\mathbf{Y}_{u}$ and $\mathbf{Y}_{b}$ respectively, with an enthalpy-based progress variable $c_{h}$

$$
\mathbf{Y}^{\prime}=\mathbf{Y}_{b} c_{h}+\mathbf{Y}_{u}\left(1-c_{h}\right)
$$


The burnt composition is taken as the products of complete stoichiometric combustion, consisting of $\mathrm{N}_{2}, \mathrm{CO}_{2}$ and $\mathrm{H}_{2} \mathrm{O}$. The progress variable $c_{Y}$ is given by

$$
c_{h}=\frac{\sum_{\alpha=1}^{n s p e c}\left[Y_{\alpha} h_{\alpha}(T)-Y_{\alpha, u} h_{\alpha}\left(T_{u}\right)\right]}{\sum_{\alpha=1}^{n s p e c}\left[Y_{\alpha, b} h_{\alpha}\left(T_{b}\right)-Y_{\alpha, u} h_{\alpha}\left(T_{u}\right)\right]},
$$

where $\mathbf{Y}$ and $T$ are the original unmodified mass fraction vector and temperature, and $h_{\alpha}$ is the specific total enthalpy of each species.

\section{A.2. Step 2}

The second step produces a flame solution using the modified composition as the inlet condition with the flame positioned $67 \mu \mathrm{m}$ from the inlet (Fig. A1 bottom). The total domain length is $200 \mu \mathrm{m}$. The flame residence time for the modified flame simulation is sufficiently small that the results are not influenced significantly by chemical reaction upstream of the modified flame front, and sufficiently large that the flame speed is not influenced significantly by diffusive flux through the domain inlet. This is confirmed in Fig. 4 by applying the two-step procedure without removing intermediate species from the reactant mixture: the flame speeds obtained using this 'reduced' solution domain closely follow the residence time-dependence obtained using a single 'full' solution domain with the same total residence time. The difference due to the use of the reduced domain procedure is negligible compared with the effect of replacing the intermediate species. This confirms the validity of the modified flame approach for determining the relative influences of intermediate species and thermal effects. 